\title{
ON THE CONTINUATION OF MEROMORPHIC FUNCTIONS
}

\author{
PENTTI JÄRVI
}

1. Let $W$ be an open Riemann surface and let $V$ be an arbitrary end of $W$, i.e., a subregion of $W$ with compact relative boundary $\partial_{W} V$. Let $M C(V)$ denote the class of meromorphic functions on $V$ which have a limit at every point of the relative Stoilow ideal boundary $\beta_{V}$ of $V$. Further, let $B V(V)$ denote the class of constants and of meromorphic functions of bounded valence on $V$, and let $M D^{*}(V)$ stand for the class of meromorphic functions with a finite spherical Dirichlet integral on $V$. As in [3] and [4], we are interested in the interrelations of these three classes on certain Riemann surfaces. In Section 2, we discuss the possibility of obtaining $B V(V) \subset M C(V)$ on Riemann surfaces whose boundaries are absolutely disconnected in the sense of Sario [13]. In Section 3, we seek conditions upon $\beta$ under which $B V(\bar{V})=M C(\bar{V})$. It turns out that $A C$-removability [4], besides the absolute disconnectivity, is just what is wanted. In Section 4 we will show that $M D^{*}(\bar{V})=B V(\bar{V}) \subset$ $M C(\bar{V})$ provided that $W$ belongs to $O_{A^{0} D}$ [12]. This result completes a theorem by Matsumoto [9].

In the course of this work we shall make some comments on [7], [8] and [11]. All of them contain invalid argumentation at certain points. We have not been able to restore corresponding results completely.

2. Let $W$ be an open Riemann surface with absolutely disconnected boundary $[13$, p. 240]. In case $W$ is a plane domain, this means that $\hat{\mathbf{C}} \backslash W(\hat{\mathbf{C}}=\mathbf{C} \cup\{\infty\})$ belongs to $N_{S B}[1$, p. 105]. An immediate consequence is that all univalent functions on $W$ can be extended to homeomorphisms of the Riemann sphere. More generally, one may expect that all meromorphic functions of bounded valence on $W$ possess a limit at every point of $\beta$, the Stoillow boundary of $W$, even if $W$ is nonplanar. Actually, Lemma 2 in [11, p. 177] asserts that this is the case for surfaces of finite genus. Unfortunately, the author overlooked serious problems related to the path lifting. We have not managed to restore her assertion. However, we will give a positive result in a special case which covers the canonical mappings treated in [11].

Proposition 1. Let $W$ be an open Riemann surface, and let $V$ be an end of $W$ such that $\beta_{V}$ is absolutely disconnected. Suppose $f$ is a meromorphic function of bounded valence on $V$ such that no $\mathrm{Cl}(f ; p)$, the cluster set of $f$ at $p \in \beta_{V}$, separates the (extended) plane. Then $f$ admits a continuous extension to $\beta_{V}$. 
Proof. Fix $p_{0} \in \beta_{V}$. We will show that $\mathrm{Cl}\left(f ; p_{0}\right)$ reduces to a singleton. Since $f$ has bounded valence, $\operatorname{Cl}\left(f ; \beta_{V}\right)=\bigcup_{p \in \beta_{V}} \operatorname{Cl}(f ; p)$ is nowhere dense in $\hat{\mathbf{C}}$; this is readily seen by a simple category argument (see e.g. [3, p. 312]). Hence we may assume, performing an auxiliary linear fractional mapping, that $f$ is bounded in $V$.

Denote by $\Gamma$ the family of rectifiable dividing cycles in $V$ which separate $p_{0}$ from $\partial_{W} V$, and let $\lambda(\Gamma)$ denote the extremal length of $\Gamma$. Since $\beta_{V}$ is absolutely disconnected, $\lambda(\Gamma)=0$ [5, Theorem 5]. Suppose, for the moment, that $\mathrm{Cl}\left(f ; p_{0}\right)$ is a proper continuum. Denote by $d$ the diameter of $\mathrm{Cl}\left(f ; p_{0}\right)$. It follows from Lemma 2 in [4, p. 44] that $l(f(c))$, the Euclidean length of $f(c)$, exceeds $d$ for every $c \in \Gamma$. The boundedness of $f(V)$ then implies that $\lambda(f(\Gamma))>0$. But this state of affairs contradicts the well-known relation

$$
\lambda(f(\Gamma)) \leqq N \lambda(\Gamma),
$$

where $N=\max \left\{v_{f}(z)=\sum_{f(p)=z} n(p ; f) \mid z \in \mathbf{C}\right\}$ and $n(p ; f)$ denotes the multiplicity of $f$ at $p$. Thus $\operatorname{Cl}\left(f ; p_{0}\right)$ is a singleton as was asserted.

Remark 1. It follows from [4, Theorem 1] that $\mathrm{Cl}\left(f ; \beta_{V}\right)$ is totally disconnected. It seems, however, that it need not be of class $N_{S B}$ (see [13, p. 289]).

Remark 2. Let $W, V$ and $\beta_{V}$ be as above, and suppose that $f$ defines a proper meromorphic mapping of $V$ onto $f(V)$, i.e., the valence function $v_{f}$ is finite and constant in $f(V)$. Then $f$ admits a continuous extension to $\beta_{V}$, and $\mathrm{Cl}\left(f ; \beta_{V}\right)$ is of class $N_{S B}$. This result is due to Jurchescu [6, Theorem 1].

Corollary. Let $W$ be an open Riemann surface of finite genus, let $V$ be an end of $W$ with $\beta_{V}$ absolutely disconnected. Suppose $f$ is a meromorphic function of bounded valence on $V$ such that each $\mathrm{Cl}(f ; p), p \in \beta_{V}$, is a line segment. Then $f$ admits a continuous extension to $\beta_{V}$. Accordingly, the conclusion holds for the parallel slit mappings discussed, e.g., in [10] and [11].

As usual, let $O_{A D}$ denote the class of Riemann surfaces which do not carry nonconstant analytic functions with a finite Dirichlet integral ( $A D$-functions). Further, let $O_{A D, n}, 1 \leqq n<\infty$, denote the class of Riemann surfaces tolerating no $A D$-function $f$ with $\max \left\{v_{f}(z) \mid z \in \mathbf{C}\right\} \leqq n$. These classes were introduced in [7, p. 381]. The authors' object was to establish the relation

$$
\left(\bigcap_{n=1}^{\infty} O_{A D, n}\right) \backslash O_{A D} \neq \emptyset .
$$

Unfortunately, their argument to this end is incorrect. A source of error is the identification of $A D$-functions with related homeomorphisms onto the Riemannian image. We will indicate how (1) can be obtained provided the assertion of Mori, cited at the outset of this section, holds true at least for planar surfaces (cf. [7, p. 381]). Let $E$ be a compact set in $\hat{\mathbf{C}}$ which belongs to $N_{S B} \backslash N_{D}$ [1, p. 105]. Then $W=\hat{\mathbf{C}} \backslash E$ does not belong to $O_{A D}$. Further, let $f$ be an $A D$-function of bounded valence on 
$W$. Then $m(f(W))$, the two-dimensional measure of $f(W)$, is finite. This implies that $\hat{\mathbf{C}} \backslash f(W)$ does not belong to $N_{S D}\left[1\right.$, p. 105]. But $N_{S D}=N_{S B}[1$, p. 116]. Therefore we can find an injective bounded analytic function $\varphi$ in $f(W)$. Then $g=\varphi \circ f$ is both bounded and of bounded valence in $W$. By our provision, $g$ admits a continuous extension $g^{*}$ to $W \cup \beta=\hat{\mathbf{C}}$. Since $g^{*}$ is bounded in $\hat{\mathbf{C}}$, we infer from [3, Lemma 3] (cf. also [4, Theorem 1]) that $v_{g}(z)=\infty$ for "most" $z \in g(W)$, i.e., $v_{f}(z)=\infty$ for "most" $z \in f(W)$. This contradiction completes the argument.

3. Suppose that $\beta$, the ideal boundary of $W$, is admissible, i.e., for each $p \in \beta$ there is an end $V \subset W$ with $p \in \beta_{V}$ such that $M C(V)$ is non-trivial [4, p. 34]. Then the $A C$-removability of $\beta[4$, p. 40] can be characterized by the condition $M C(\bar{V}) \subset$ $B V(\bar{V})$ for each end $V \subset W[4$, Theorems 1 and 2]. Thus, one may ask what more is needed to insure $M C(\bar{V})=B V(\bar{V})$ for each end $V \subset W$. Theorem 9 in [3, p. 311] suggests that the absolute disconnectivity of $\beta$ would do the job. This is the case as shown by

Theorem 1. Let $W$ be an open Riemann surface with admissible ideal boundary $\beta$. Suppose $\beta$ is absolutely disconnected and $A C$-removable. Then $M C(\bar{V})=B V(\bar{V})=$ $M D^{*}(\bar{V})$ for each end $V \subset W$. Conversely, if $M C(\bar{V})=B V(\bar{V})$ for each end $V \subset W$, then $\beta$ is absolutely disconnected and $A C$-removable.

Proof. Suppose $\beta$ is absolutely disconnected and $A C$-removable. As noted before, this implies $M C(\bar{V}) \subset B V(\bar{V})$ for each end $V \subset W$. To establish the reverse inclusion, let $V$ be an end of $W$ and let $f \in B V(\bar{V})$. Fix $p_{0} \in \beta_{V}$ and choose a subend $V^{\prime}$ of $V$ such that $p_{0} \in \beta_{V^{\prime}}$ and $M C\left(\bar{V}^{\prime}\right)$ contains a nonconstant function $f_{0}$. Reducing $V^{\prime}$ and performing a preliminary linear fractional mapping, we may assume that $f_{0}$ is bounded in $V^{\prime}$. Let $f_{0}^{*}$ stand for the extension of $f_{0}$ to $\beta_{V^{\prime}}$. Since $\beta_{V^{\prime}}$ is $A C$-removable, $f_{0}^{*}\left(\beta_{V^{\prime}}\right)$ is of class $N_{C}^{\prime}\left[4\right.$, p. 38]. We are going to show that $f_{0}^{*}\left(\beta_{V^{\prime}}\right) \in N_{D}$ also.

Since $f_{0}^{*}\left(\beta_{V^{\prime}}\right)$ is totally disconnected, we may arrange $f_{0}^{*}\left(\beta_{V^{\prime}}\right) \cap f_{0}\left(\partial_{W} V^{\prime}\right)=\emptyset$. For $z \in \mathbf{C} \backslash f_{0}\left(\partial_{W} V^{\prime}\right)$, let $i\left(z ; f_{0}\left(\partial_{W} V^{\prime}\right)\right)$ denote the index of $z$ with respect to $f_{0}\left(\partial_{W} V^{\prime}\right)$ [4, p. 34]. By [4, Lemma 1], $v_{f_{0}}(z)<i\left(z ; f_{0}\left(\partial_{W} V^{\prime}\right)\right)$ for each $z \in f_{0}^{*}\left(\beta_{V^{\prime}}\right)$. Set $n=\max \left\{i\left(z ; f_{0}\left(\partial_{W} V^{\prime}\right)\right) \mid z \in \mathbf{C}\right\}$ and $E_{i}=\left\{z \in f_{0}^{*}\left(\beta_{V^{\prime}}\right) \mid v_{f_{0}}(z) \leqq i\right\}$. Of course, $E_{n-1}=f_{0}^{*}\left(\beta_{V^{\prime}}\right)$. Assume that $E_{0}$ is not of class $N_{D}$. Since $E_{0}$ belongs to $N_{C}^{\prime}$, it also fails to be of class $N_{S B}$. Therefore, we can find a nonweak boundary point, say $z_{0}$, of the planar surface $\hat{\mathbf{C}} \backslash E_{0}\left[13\right.$, p. 152 and p. 239]. Pick out a point $p$ in $\left(f_{0}^{*}\right)^{-1}\left(z_{0}\right)$ and denote by $n_{0}$ the local degree of $f_{0}^{*}$ at $p$ (see [4, p. 35]). Next choose a Jordan domain $D \subset \mathbf{C}$ such that $\partial D \cap f_{0}^{*}\left(\beta_{V^{\prime}}\right)=\emptyset$ and $n_{0}=$ the constant valence of $f_{0} \mid U$ in $D \backslash E_{n-1}$, where $U$ denotes the component of $f_{0}^{-1}(D)$ with $p \in \beta_{U}$ (cf. [3, p. 306]).

By [5, Theorem 5], we can find a nonnegative Borel function $\varrho$ on $D \backslash E_{0}$ and a positive $\delta$ such that

$$
\iint_{D \backslash E_{0}} \varrho^{2} d x d y<\infty \text { and } \int_{c} \varrho|d z| \geqq \delta
$$


for each rectifiable Jordan curve $c \subset D \backslash E_{0}$ separating $z_{0}$ from $\partial D$. Denote by $\hat{\varrho}(z)|d z|$ the pullback to $U$ via $f_{0}$ of the conformal metric $\varrho(z)|d z|$ on $D \backslash E_{0}$. Since the valence of $f_{0} \mid U$ is bounded by $n_{0}$, we have

$$
\iint_{U} \hat{\varrho}^{2} d x d y<\infty .
$$

By the weakness of $p_{0}$, we can find a rectifiable dividing cycle $c^{\prime}$ in $U$ separating $p_{0}$ from $\partial_{W} U$ such that

$$
\int_{c^{\prime}} \hat{\varrho}|d z|<\delta
$$

[5, Theorem 5], [13, Theorem IV 2 C]. Then $c^{\prime}$ is homologous to $\partial_{W} U^{\prime}$ for some subend $U^{\prime}$ of $U$ with $p_{0} \in \beta_{U^{\prime}}\left[13\right.$, p. 84]. It follows that $i\left(z_{0} ; f_{0}\left(c^{\prime}\right)\right)=n_{0}$. Clearly $f\left(c^{\prime}\right)$ contains a rectifiable Jordan curve $c \subset D \backslash E_{0}$ which separates $z_{0}$ from $\partial D$. Of course,

$$
\int_{c} \varrho|d z| \leqq \int_{f\left(c^{\prime}\right)} \varrho|d z|=\int_{c^{\prime}} \hat{\varrho}|d z|<\delta .
$$

This contradiction to (2) proves $E_{0} \in N_{D}$.

Next suppose that, for some $i, E_{i}$ is of class $N_{D}$ and fix $z_{0} \in E_{i+1} \backslash E_{i}$. Choose a neighbourhood $D$ of $z_{0}$ such that $\partial D$ is a Jordan curve with $\partial D \cap\left(E_{n-1} \cup f_{0}\left(\partial_{W} V^{\prime}\right)\right)=\emptyset$ and $f_{0}^{-1}(D)$ contains $j(j \leqq i+1)$ relatively compact mutually disjoint Jordan regions $V_{k}$ in $V^{\prime}$ such that each $z \in D$ has exactly $i+1$ pre-images (with due account of multiplicities) in $\bigcup_{k=1}^{j} V_{k}$. Then $f_{0} \mid V^{\prime} \backslash \bigcup_{k=1}^{j} V_{k}$ assumes no value in $E_{i+1} \cap D$. Thus we have the state of affairs treated above. Hence a reproduction of the preceding arguments yields $E_{i+1} \cap D \in N_{D}$. Further, [12, Theorem VI $\left.1 \mathrm{~L}\right]$ in conjunction with the Lindelöf covering theorem gives $E_{i+1} \in N_{D}$. It follows that $E_{n-1}=f_{0}^{*}\left(\beta_{V^{\prime}}\right)$ is of class $N_{D}$ as was asserted.

Let $G$ denote the component of $\mathbf{C} \backslash f_{0}\left(\partial_{W} V^{\prime}\right)$ which contains $f_{0}^{*}\left(p_{0}\right)$. Let $n$ stand for the constant value of $v_{f_{0}}(z)$ in $G \backslash f_{0}^{*}\left(\beta_{V^{\prime}}\right)$. Then $f$ satisfies in $f_{0}^{-1}\left(G \backslash f_{0}^{*}\left(\beta_{V^{\prime}}\right)\right)$ an identity

$$
f^{n}+\sum_{i=1}^{n}\left(a_{i} \circ f_{0}\right) f^{n-i}=0,
$$

where $a_{1}, \ldots, a_{n}$ are meromorphic functions on $G \backslash f_{0}^{*}\left(\beta_{V^{\prime}}\right)$. Making use of the relation $f \in B V\left(\bar{V}^{\prime}\right)$ and arguing as in [2, pp. 14 and 18], it can be shown that, for every $i, a_{i}$ admits a meromorphic extension over $f_{0}^{*}\left(\beta_{V^{\prime}}\right) \cap G$. Thus we may regard each $a_{i}$ as defined everywhere in $G$. Denote by $\tilde{G}$ the Riemann surface of the relation

$$
P(z, w)=w^{n}+\sum_{i=1}^{n} a_{i}(z) w^{n-i}=0, \quad z \in G,
$$

(note that $\widetilde{G}$ is a finite union of connected Riemann surfaces). By an argument involving $\widetilde{G}$ and the center and value mappings associated with (3), it can be shown (cf. again the proof of $\left[2\right.$, Theorem 1]) that $\mathrm{Cl}\left(f ;\left(f_{0}^{*}\right)^{-1}\left(f_{0}^{*}\left(\beta_{V^{\prime}}\right) \cap G\right)\right)$ belongs to $N_{D}$. In particular, $\mathrm{Cl}\left(f ; p_{0}\right)$ reduces to a singleton, i.e., $f$ admits a continuous extension to $p_{0}$. Thus $B V(\bar{V}) \subset M C(\bar{V})$. 
The relation $B V(\bar{V}) \subset M D^{*}(\bar{V})$ being trivial, it remains to show that $M D^{*}(\bar{V}) \subset$ $B V(\bar{V})$ for each end $V \subset W$. So let $f \in M D^{*}(\bar{V})$ be nonconstant. Since the problem is of a local nature, we may assume that $\partial_{W} V$ is piecewise analytic and $M C(\bar{V})$ contains a bounded nonconstant function $f_{0}$. By virtue of $f \in M D^{*}(\bar{V})$, we may also assume that $f$ omits in $\bar{V}$ a compact set $E \subset \mathbf{C}$ with $m(E)>0$. Then, by an important theorem of Nguyen Xuan Uy [14, Theorem 4.1], we can find a nonconstant analytic function $g$ such that both $g$ and $g^{\prime}$ are bounded in $\hat{\mathbf{C}} \backslash E$. We will show that $h=g \circ f \in A D(\bar{V})$. To this end, choose $R>0$ such that $E \subset D(0, R)=\{z \in \mathbf{C}|| z \mid<R\}$. Set $F_{1}=f^{-1}(D(0, R)), F_{2}=f^{-1}(\hat{\mathbf{C}} \backslash D(0, R))$ and choose $M>0$ such that $\left|g^{\prime}(z)\right| \leqq M$ for $z \in \hat{\mathbf{C}} \backslash E$. Obviously

$$
\begin{aligned}
\iint_{F_{1}} d h \wedge * d \bar{h}= & \iint_{F_{1}}\left|g^{\prime}(f(p))\right|^{2} d f \wedge * d \bar{f} \leqq\left(1+R^{2}\right)^{2} M^{2} \iint_{F_{1}} \frac{1}{\left(1+R^{2}\right)^{2}} d f \wedge * d \bar{f} \\
& \leqq\left(1+R^{2}\right)^{2} M^{2} \iint_{F_{1}} \frac{1}{\left(1+|f(p)|^{2}\right)^{2}} d f \wedge * d \bar{f}<\infty .
\end{aligned}
$$

Let $\varphi$ denote the mapping $z \mapsto 1 / z, \quad z \in \hat{\mathbf{C}} \backslash D(0, R)$. Then $g \mid \hat{\mathbf{C}} \backslash D(0, R)=g_{1} \circ \varphi$ with $g_{1}$ analytic in $\overline{D(0,1 / R)}$. Suppose $\left|g_{1}^{\prime}(z)\right| \leqq M_{1}$ for $z \in \overline{D(0,1 / R)}$. Then

$$
\begin{gathered}
\iint_{F_{2}} d h \wedge * d \bar{h}=\iint_{F_{2}}\left|g_{1}^{\prime}(\varphi(f(p)))\right|^{2} d(\varphi \circ f) \wedge * d(\overline{\varphi \circ f}) \\
\leqq M_{1}^{2} \iint_{F_{2}} d(\varphi \circ f) \wedge * d(\overline{\varphi \circ f}) \\
\leqq M_{1}^{2}\left(1+\left(\frac{1}{R}\right)^{2}\right)^{2} \iint_{F_{2}} \frac{1}{\left(1+|\varphi(f(p))|^{2}\right)^{2}} d(\varphi \circ f) \wedge * d \overline{(\overline{\varphi \circ f})} \\
=M_{1}^{2}\left(1+\left(\frac{1}{R}\right)^{2}\right)^{2} \iint_{F_{2}} \frac{1}{\left(1+|f(p)|^{2}\right)^{2}} d f \wedge * d \bar{f}<\infty .
\end{gathered}
$$

The assertion follows.

Let $G$ denote a component of $\mathbf{C} \backslash f_{0}\left(\partial_{W} V\right)$ with $G \cap f_{0}^{*}\left(\beta_{V}\right) \neq \emptyset$, and let $n$ denote the constant value of $v_{f_{0}}(z)$ in $G \backslash f_{0}^{*}\left(\beta_{V}\right)$. Then $h$ satisfies in $f_{0}^{-1}\left(G \backslash f_{0}^{*}\left(\beta_{V}\right)\right)$ an identity

$$
h^{n}+\sum_{i=1}^{n}\left(a_{i} \circ f_{0}\right) h^{n-i}=0
$$

with $a_{i}$ analytic in $G \backslash f_{0}^{*}\left(\beta_{V}\right)$. As shown before, we have $f_{0}^{*}\left(\beta_{V}\right) \in N_{D}$. Because $h$ is both bounded and Dirichlet bounded, each $a_{i}$ admits an analytic extension over $f_{0}^{*}\left(\beta_{V}\right) \cap G$ (cf. again [2, pp. 14-15 and p. 23]). As before, these extensions permit us to conclude that $\mathrm{Cl}\left(h ;\left(f_{0}^{*}\right)^{-1}\left(f_{0}^{*}\left(\beta_{V}\right) \cap G\right)\right)$ belongs to $N_{D}$. It follows that $\mathrm{Cl}\left(h ; \beta_{V}\right)$ is totally disconnected. Hence by [4, Lemma 2], $h$ has bounded valence. Consequently, the same is true of $f$, i.e., $f \in B V(\bar{V})$. This completes the first part of the proof.

Next suppose that $M C(\bar{V})=B V(\bar{V})$ for each end $V \subset W$. It is immediate by [4, Theorems 1 and 2] that $\beta$ is $A C$-removable. Fix an arbitrary $p_{0} \in \beta$. It remains to prove that $p_{0}$ is weak. So choose an end $V \subset W$ such that $p_{0} \in \beta_{V}$ and $M C(\bar{V})$ 
contains a nonconstant bounded function $f_{0}$. Since $f_{0}^{*}\left(\beta_{V}\right)$ is of class $N_{C}^{\prime}[4$, p. 40], we may assume that $f_{0}^{*}\left(\beta_{V}\right) \cap f_{0}\left(\partial_{W} V\right)=\emptyset$. In order to prove that $f_{0}^{*}\left(\beta_{V}\right)$ is of class $N_{S B}$, we argue as in the first part of the proof.

Let $G$ denote the component of $\mathbf{C} \backslash f_{0}\left(\partial_{W} V\right)$ which contains $f_{0}^{*}\left(p_{0}\right)$, and let $n$ denote the constant value of $v_{f_{0}}(z)$ in $G \backslash f_{0}^{*}\left(\beta_{V}\right)$. Set $E_{i}=\left\{z \in f_{0}^{*}\left(\beta_{V}\right) \cap G \mid v_{f_{0}}(z) \leqq i\right\}$, $i=0, \ldots, n-1$. Suppose for the moment that $E_{0}$ fails to be of class $N_{S B}$. Then $E_{0}$ contains a nonweak boundary point, say $z_{0}$, of $\hat{\mathbf{C}} \backslash E_{0}$. Pick out a point $p \in\left(f_{0}^{*}\right)^{-1}\left(z_{0}\right)$. By [13, p. 152], we can find a univalent mapping $\varphi$ of $\hat{\mathbf{C}} \backslash E_{0}$ into $\hat{\mathbf{C}}$ such that $\mathrm{Cl}\left(\varphi ; z_{0}\right)$ is a proper continuum. Given any subend $V^{\prime}$ of $V$ with $p \in \beta_{V^{\prime}}$, there is an open neighborhood $D$ of $z_{0}$ such that $D \backslash f_{0}^{*}\left(\beta_{V}\right) \subset f_{0}\left(V^{\prime}\right)$ [4, Lemma 1]. We conclude that $\mathrm{Cl}\left(\varphi \circ f_{0} ; p\right)$ is also a proper continuum. Hence $\varphi \circ f_{0}$, although a member of $B V(\bar{V})$, does not admit a continuous extension to $p$. Thus $E_{0}$ is of class $N_{S B}$ and, by virtue of $E_{0} \in N_{C}^{\prime}$, even of class $N_{D}$.

Now suppose that, for some $i, E_{i}$ is of class $N_{D}$ and fix $z_{0} \in E_{i+1} \backslash E_{i}$. The argument given in the proof of $B V(\bar{V}) \subset M C(\bar{V})$ reduces the situation to that discussed above. It follows that $E_{i+1} \in N_{D}$. Altogether, $E_{n-1}=f_{0}^{*}\left(\beta_{V}\right) \cap G$ is of class $N_{S B}$. Let $U$ denote the component of $f_{0}^{-1}(G)$ with $p_{0} \in \beta_{U}$. We infer from [13, Theorem $\mathrm{X} 4 \mathrm{~F}$ ] or [6, Theorem 1] that $\beta_{U}$ is absolutely disconnected. In particular, $p_{0}$ is weak. The proof is complete.

Remark. Recall that the union of two sets of class $N_{S B}$ need not belong to $N_{S B}\left[13\right.$, p. 289]. This fact explains the role of the class $N_{D}$ in the last part of the proof.

The argument used in the proof of the relation $M D^{*}(\bar{V}) \subset B V(\bar{V})$ obviously yields the following removability result, which seems to be new.

Theorem 2. Let $G$ be a plane domain and let $E \subset G$ be a compact set of class $N_{D}$. Then every meromorphic function with a finite spherical Dirichlet integral in $G \backslash E$ can be extended to a meromorphic function in $G$. In particular, every meromorphic function with a finite spherical Dirichlet integral in $\hat{\mathbf{C}} \backslash E$ is the restriction to $\hat{\mathbf{C}} \backslash E$ of a rational function.

As mentioned before, $M C(\bar{V}) \subset B V(\bar{V})$ for every end $V \subset W$ whenever the ideal boundary of $W$ is $A C$-removable. This statement can be generalized as follows.

Proposition 2. Let $W$ and $W^{\prime}$ be Riemann surfaces with ideal boundaries $\beta$ and $\beta^{\prime}$, respectively. Suppose that every point of $\beta$ is AC-removable whenever it is admissible. Let $f$ be a nonconstant analytic mapping of $W$ into $W^{\prime}$ which admits a continuous extension $f^{*}: W \cup \beta \rightarrow W^{\prime} \cup \beta^{\prime}$. Then $f$ has bounded valence.

Proof. Let $\beta_{1}$ denote the set $\left\{p \in \beta \mid f^{*}(p) \in W^{\prime}\right\}$. Clearly $\beta_{1}$ is a relatively open subset of $\beta$. For each $p \in \beta_{1}$, choose an end $V_{p} \subset W$ such that $p \in \beta_{V_{p}}$ and $f^{*}\left(\bar{V}_{p} \cup \beta_{V_{p}}\right) \subset W^{\prime}$. By assumption and [4, Theorem 2], $f^{*}\left(\beta_{V_{p}}\right)$ is a totally disconnected subset of $W^{\prime}$. Thanks to Lindelöf, we can select from the covering $\left\{V_{p} \cup \beta_{V_{p}} \mid p \in \beta_{1}\right\}$ of $\beta_{1}$ a countable subcovering $\left\{V_{n} \cup \beta_{V_{n}} \mid n \in N\right\}$. Thus $f^{*}\left(\beta_{1}\right)=$ 
$\cup_{n=1}^{\infty} f^{*}\left(\beta_{V_{n}}\right)$ is a closed and totally disconnected subset of $W^{\prime}$. It follows that $v_{f}$, the valence function of $f$, is finite and constant, say $m$, in $W^{\wedge} \backslash f^{*}\left(\beta_{1}\right)$. By the lower semicontinuity of $v_{f}, v_{f}(q) \leqq m$ for every $q \in W^{\prime}$.

Now suppose that $W$ is parabolic. Then every admissible boundary point of $W$ is $A C$-removable [4, p. 40]. Hence we have immediately the following

Corollary. Let $W$ and $W^{\prime}$ be parabolic Riemann surfaces with ideal boundaries $\beta$ and $\beta^{\prime}$, respectively. Let $f$ be a nonconstant analytic mapping of $W$ into $W^{\prime}$ which admits a continuous extension $f^{*}: W \cup \beta \rightarrow W^{\prime} \cup \beta^{\prime}$. Then $f$ has bounded valence.

Remark. This result is contained in [8, Theorem 1]. However, the proof there relies on the incorrect claim that the Stoillow boundary is a countable set.

4. Next suppose that $W$ belongs to $O_{A^{0} D}[12$, p. 17] and $V$ is an end of $W$. Then, by a result of Matsumoto and Kuroda [12, p. 372], every function $f \in M D^{*}(\bar{V})$ has the localizable Iversen property [12, p. 365]. By Stoillow's principle on Inversen's property [12, p. 370], $\mathrm{Cl}\left(f ; \beta_{V}\right)$ is either total, i.e., $\mathrm{Cl}\left(f ; \beta_{V}\right)=\hat{\mathbf{C}}$, or $\mathrm{Cl}\left(f ; \beta_{V}\right)$ is totally disconnected. It was shown by Matsumoto [9, Theorem 3], [12, p. 373] that for $f \in A D(\bar{V})$, only the latter alternative can occur, i.e., every function in $A D(\bar{V})$ admits a continuous extension to $\beta_{V}$. Actually, this is the case with all $M D^{*}$ functions as shown by

Theorem 3. Let $W$ be an open Riemann surface of class $O_{A^{0} D}$. Then $B V(\bar{V})=$ $M D^{*}(\bar{V}) \subset M C(\bar{V})$ for each end $V \subset W$.

Proof. Let $V \subset W$ be an end, and suppose that $f \in M D^{*}(\bar{V})$ is nonconstant. To prove that $f \in B V(\bar{V})$, it clearly suffices to show that for each $p \in \beta_{V}$ there is a subend $V^{\prime}$ of $V$ such that $p \in \beta_{V^{\prime}}$ and $f \mid \bar{V}^{\prime}$ has bounded valence. So fix $p \in \beta_{V}$ and choose a subend $V^{\prime}$ of $V$ such that $p \in \beta_{V^{\prime}}$ and $f$ omits in $\bar{V}^{\prime}$ a compact set $E \subset \mathbf{C}$ with $m(E)>0$. As in the proof of Theorem 1, we can find a nonconstant analytic function $g$ in $\hat{\mathbf{C}} \backslash E$ such that $h=g \circ\left(f \mid \bar{V}^{\prime}\right) \in A D\left(\bar{V}^{\prime}\right)$. By the result of Matsumoto cited previously, $\mathrm{Cl}\left(h ; \beta_{V^{\prime}}\right)$ is totally disconnected. This implies $([4$, Lemma 1] or $\left[12\right.$, p. 370]) that $h$ has bounded valence. Thus $f \mid \bar{V}^{\prime}$ has bounded valence, too. We conclude that $f \in B V(\bar{V})$. That $f$ also belongs to $M C(\bar{V})$ now follows readily from Stoïlow's principle on Iversen's property. Indeed, provided $\mathrm{Cl}\left(f ; \beta_{V}\right)$ is total, a standard argument involving the Baire category (see, e.g., [3, Lemma 3]) gives $f \notin B V(\bar{V})$. Hence $\mathrm{Cl}\left(f ; \beta_{V}\right)$ is totally disconnected, whence $f \in M C(\bar{V})$.

Remark. It is readily verified that $\operatorname{Cl}\left(f ; \beta_{V}\right)=f^{*}\left(\beta_{V}\right)$ is of class $N_{D}$ : just decompose $f^{*}\left(\beta_{V}\right)$ by means of the valence function $v_{f}$ as in the proof of Theorem 1 and apply [12, Theorem VI 2 C]. It seems that for $f \in B V(\bar{V})$ this result has also been obtained by Qiu Shuxi (see [15, pp. 152-3]). The relation $f^{*}\left(\beta_{V}\right) \in N_{D}$ can be used to show that $B V(\bar{V})=M D^{*}(\bar{V})$ constitutes a field (see the proofs of [2, Theorem 7] and [3, Theorem 5]). 
Let $U_{S}$ denote the class of open Riemann surfaces whose ideal boundary contains a point of positive harmonic measure [12, p. 385]. Further, let $O_{M D^{*}}$ denote the class of Riemann surfaces which do not carry nonconstant meromorphic functions with a finite spherical Dirichlet integral. Of course, $O_{M D^{*}} \subset O_{A D}$. By [13, Theorem $X 4 \mathrm{C}$ ] we immediately obtain the following corollary, which sharpens another result by Matsumoto [9, Theorem 4], [12, Theorem VI 5 B].

Corollary. Suppose $W \in U_{S} \cap O_{A^{0} D}$ and let $K$ be an arbitrary compact set in $W$ with connected complement. Then $W \backslash K \in O_{M D^{*}}$.

\section{References}

[1] Ahlfors, L., and A. BeurLing: Conformal invariants and function-theoretic null-sets. Acta Math. 83, 1950, 101-129.

[2] JärVI, P.: Removability theorems for meromorphic functions. - Ann. Acad. Sci. Fenn. Ser. A I Math. Dissertationes 12, 1977, 1-33.

[3] JÄRVI, P.: Meromorphic functions on certain Riemann surfaces with small boundary. - Ann. Acad. Sci. Fenn. Ser. A I Math. 5, 1980, 301-315.

[4] JÄRVI, P.: On meromorphic functions continuous on the Stoilow boundary. - Ann. Acad. Sci. Fenn. Ser. A I Math. 9, 1984, 33-48.

[5] JuRchescu, M.: Modulus of a boundary component. - Pacific J. Math. 8, 1958, 791-809.

[6] JURChescu, M.: A maximal Riemann surface. - Nagoya Math. J. 20, 1962, 91-93.

[7] KUSUNOKI, Y., and M. TANIGUCHI: Remarks on Fuchsian groups associated with open Riemann surfaces. - Riemann surfaces and related topics: Proceedings of the 1978 Stony Brook conference, edited by I. Kra and B. Maskit. Annals of Mathematics Sudies 97. Princeton University Press, Princeton, New Jersey, 1981, 377-390.

[8] LAINE, I.: Quasirational mappings on parabolic Riemann surfaces. - Ann. Acad. Sci. Fenn. Ser. A I Math. 482, 1970, 1-26.

[9] Matsumoto, K.: Analytic functions on some Riemann surfaces II. - Nagoya Math. J. 23, 1963, 153-164.

[10] Mizumoto, H.: Theory of Abelian differentials and relative extremal length with applications to extremal slit mappings. - Japan. J. Math. 37, 1968, 1-58.

[11] Mor, M.: Canonical conformal mappings of open Riemann surfaces. - J. Math. Kyoto Univ. 3, 1964, 169-192.

[12] SARTo, L., and M. NAKAI: Classification theory of Riemann surfaces. - Die Grundlehren der mathematischen Wissenschaften 164, Springer-Verlag, Berlin-Heidelberg-New York, 1970.

[13] SARIO, L., and K. OIKAWA: Capacity functions. - Die Grundlehren der mathematischen Wissenschaften 149, Springer-Verlag, Berlin-Heidelberg-New York, 1969.

[14] UY, N. X.: Removable sets of analytic functions satisfying a Lipschitz condition. - Ark. Mat. $17,1979,19-27$.

[15] ZhaNG, M.-Y.: Riemann surfaces. - Analytic functions of one complex variable. Contemp. Math. 48. American Mathematical Society, Providence, R. I., 1985.

University of Helsinki

Department of Mathematics

SF-00100 Helsinki 10

Finland

Received 29 April 1986 